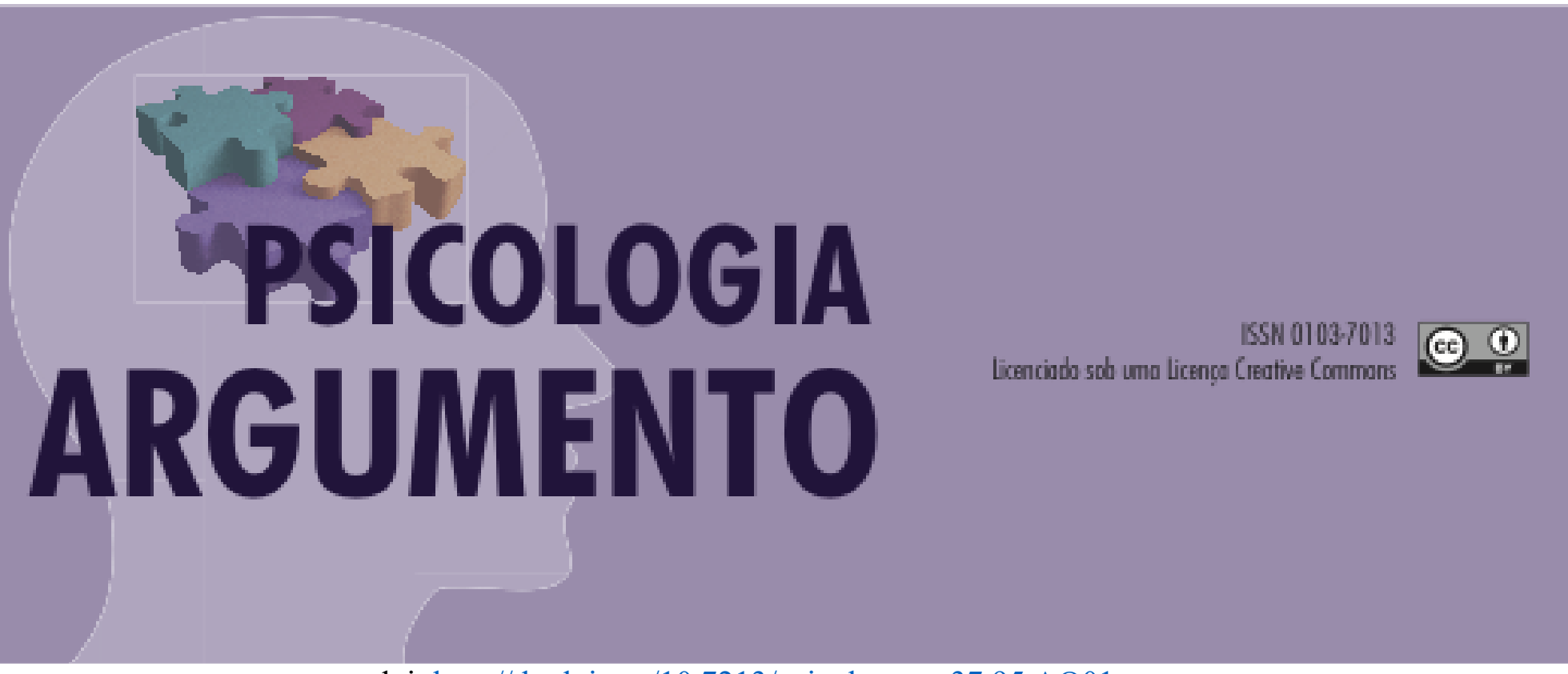

doi: http://dx.doi.org/10.7213/psicolargum.37.95.AO01

\title{
O que o Eletroencefalograma tem a nos dizer sobre a experiência religiosa: uma revisão sistemática
}

What Electroencephalogram has to tell us about religious experience: a systematic review

Qué nos dice el electroencefalograma sobre la experiencia religiosa: una revisión sistemática

\author{
Matheus Svóboda Caruzo \\ Mestrando em Psicologia pela Universidade Federal Rural do Rio de Janeiro \\ (PPGPSI/UFRRJ), Brasil. Email:psicaruzo@gmail.com \\ https://orcid.org/0000-0002-4319-5237 \\ Emmy Uehara Pires \\ http://orcid.org/0000-0002-3845-4839 \\ Doutorado em Psicologia pela Pontifícia Universidade Católica do Rio de Janeiro, \\ Brasil (2014). Professora Adjunta da Universidade Federal Rural do Rio de Janeiro \\ (UFRRJ), Brasil. Email: emmy.uehara@gmail.com
}

\begin{abstract}
Resumo
É possível observar historicamente o antagonismo delineado entre ciência e religião. Desde Galileu e a revolução científica, foram múltiplas as disciplinas que destinaram seus anseios teóricos para o entendimento dessa interface, a exemplo das neurociências, a partir do século XIX. Atualmente, observa-se um crescente interesse em investigar os correlatos neuronais da experiência religiosa e sua implicação na vida cotidiana. O presente manuscrito apresenta uma revisão sistemática PRISMA dos estudos que buscaram compreender os correlatos neurológicos da experiência religiosa a partir da técnica Eletroencefalograma (EEG) nos últimos dezoitos anos. Para isto, a coleta de dados foi realizada nos bancos de dados Pubmed, PsycINFO, Scielo e
\end{abstract}


Science Direct. Dentre os resultados, a modulação das ondas Gama foi observada nos rituais cristãos e budistas, as ondas Teta apresentaram aumento no Cristianismo e diminuição no Espiritismo e as ondas Alfa nos rituais islâmicos. Os pesquisadores sugerem que esses resultados podem estar correlacionados com maior senso de propósito e significado de vida, bem como amplificação da empatia e compaixão.

Palavras-chave: religião, eletroencefalograma, neurociência, neuropsicologia, revisão sistemática.

\begin{abstract}
It is possible to observe historically the antagonism outlined between science and religion. From Galileu and the scientific revolution, there were multiple disciplines that aimed their theoretical aspirations for the understanding of this interface, like the neurosciences in the 19th century. There is now a growing interest in investigating the neuronal correlates of religious experience and its implication in everyday life. This manuscript presents a PRISMA systematic review of the studies that sought to understand the neurological correlates of religious experience using the Electroencephalogram (EEG) technique in the last eighteen years. For this, data collection was performed in Pubmed, PsycINFO, Scielo and Science Direct databases. Among the results, Gamma wave modulation was observed in Christian and Buddhist rituals, Theta waves showed an increase in Christianity and a decrease in Spiritualism and Alpha waves in Islamic rituals. The researchers suggest that these findings may be correlated with a greater sense of purpose and meaning in life, as well as amplification of empathy and compassion.
\end{abstract}

Keywords: religion, electroencephalogram, neurosciences, neuropsychology, systematic review

\title{
Resumen
}

Es posible observar históricamente el antagonismo esbozado entre ciencia y religión. Desde Galileu y la revolución científica, múltiples disciplinas apuntaron sus aspiraciones teóricas para la comprensión de esta interfaz, como las neurociencias en el siglo XIX. Ahora hay un interés creciente en investigar los correlatos neuronales de la experiencia religiosa y su implicación en la vida cotidiana. Este manuscrito presenta una revisión sistemática PRISMA de los estudios que buscarán comprender los correlatos neurológicos de la experiencia religiosa utilizando la técnica de electroencefalograma (EEG) en los últimos dieciocho años. Para esto, la recolección de datos se realizó en las bases de datos Pubmed, PsycINFO, Scielo y Science Direct. Entre los resultados, la modulación de la onda gamma se detecta en los rituales cristianos y budistas, las ondas Theta tiene un aumento en el cristianismo y una disminución en el espiritualismo y las ondas alfa en los rituales islámicos. Los investigadores dicen que estos hallazgos pueden estar correlacionados con un mayor sentido de propósito y significado en la vida, así como con la amplificación de la empatía y la compasión.

Palabras clave: religión, electroencefalograma, neurociencias, neuropsicología, revisión sistemática.

\section{Introdução}

Desde a revolução científica, religião e ciência são campos comumente marcados por uma distinção no imaginário social. Dentro de suas perspectivas conceituais, ambas se mostram dispostas a fundamentar a relevância da explicação que atribuem às coisas e, por vezes, a universalidade que se aplica a esta. Por conseguinte, é possível analisar historicamente a polarização facultada por entusiastas tanto das ciências quanto dos 
conhecimentos sacros (Cescon, 2011). Ainda assim, foram múltiplas as áreas que reservaram parte de seus anseios temáticos para teorizações que objetivassem um entendimento a respeito das milenares experiências e processos religiosos, como por exemplo, os estudos do alemão Friedrich Muller, entre as décadas de 1850 e 1870 (Valle, 2001).

Uma das áreas da qual emergiu a pretensão de compreender os fenômenos religiosos foi a Neurociência. Conforme evoluiu, promoveu avanços nos rumos das ciências biomédicas, humanas e sociais. Diversas pesquisas entraram em curso e desenvolveram-se técnicas precursoras de escaneamento e imageamento cerebral. $\mathrm{O}$ preâmbulo das tentativas de entender os estados mentais decorrentes da experimentação religiosa sob a ótica psicobiológica é demarcado pelas associações entre religiosidade e epilepsia do lobo temporal, abordadas nos anos derradeiros do século XIX. Como ato inaugural das pesquisas de intersecção Neurociência e religião, podemos citar os estudos de Persinger (1987), que testou a hipótese mantida viva pela Escola de Geschwind de descargas elétricas de neurônios do córtex temporal serem responsáveis pela gênese da experiência religiosa.

Atualmente, observa-se um crescente interesse em compreender os correlatos neuronais da experiência religiosa e sua implicação na vida cotidiana. Beauregard e Paquete (2008) definem experiência religiosa como um senso de união com o divino. Ainda, a caracterizam como o sentimento de ter alcançado certa elevação da realidade, a experiência de perda do senso de tempo e espaço e os sentimentos de união com a humanidade, paz e amor incondicional. É importante, ainda, ressaltar a disparidade conceitual entre termos como espiritualidade e experiência religiosa, haja vista a compreensão da religião e da espiritualidade como dimensões desassociadas.

Dentre os métodos investigativos desta interface, o Eletroencefalograma (EEG) é um dos mais proeminentes e conhecidos. Em contextos clínicos, o EEG realiza a captura e gravação da atividade elétrica espontânea do cérebro, sendo registrada a partir de múltiplos eletrodos organizados sobre o couro cabeludo. De acordo com Travis e Shear (2010), as ondas cerebrais são produzidas pelo somatório das interações elétricas dos bilhões de neurônios do cérebro, sendo mensuradas em ciclos por segundo ou Hertz (Hz). Assim, cada pessoa tem suas próprias características de ação das ondas cerebrais, isto é, cada pessoa tem uma configuração de atividade neuronal elétrica única.

Existem cinco ritmos de ondas cerebrais: Gama, Beta, Alfa, Teta e Delta, sendo a predominância de um padrão sobre os outros a responsável por determinar o nosso estado 
de consciência (Travis e Shear, 2010). Cada frequência é observada majoritariamente em áreas cerebrais específicas, contudo, estudos apontam a variabilidade de regiões ativadas no processamento de cada uma delas. Dentre os motivos para este método ser um dos mais amplamente utilizados em laboratórios médicos e psicológicos, destaca-se seu caráter não invasivo de monitoramento da atividade do cérebro.

Durante o processamento de informações, diversas áreas funcionam paralelamente. Ritmos de baixa freqüência (Alfa e Teta) engendram o sistema atencional e a memória de trabalho/operacional, caracterizando-se pelo processamento descendente ou top-down (nível de processamento mais alto influenciando o mais baixo). Em contrapartida, ritmos de maior freqüência (Beta e Gama) são modulados pelo processamento da experiência. Em um estado equipendente, tem-se o agrupamento funcional de todas as freqüências, que trabalham em conjunto (Varela, Lachaux, Rodriguez \& Martinerie, 2001).

O ritmo Gama é o que atinge frequências entre $30 \mathrm{~Hz}$ e $70 \mathrm{~Hz}$, sendo a banda de maior onda de frequência. Participa do processamento de estímulos visuais, táteis e auditivos, sendo articulado pelo processamento sensorial cruzado. Além disso, é também responsável pelo reconhecimento e reconstrução da experiência. Ao passo que se elevam as ondas Gama, mais palpável torna-se a acomodação de informação na memória de curto prazo, assim como se torna mais fácil lembrar-se de algo que havia sido esquecido. Recentemente, a freqüência Gama vem sendo associada à meditação, significada empiricamente como "um estado de amor profundo e universal" (Lutz, Rawlings, Ricard \& Davidson, 2004).

As ondas Beta atuam nos estados de vigília e percepção do tempo e espaço, nos quais o sistema atencional é integralmente requerido, atingindo freqüências de $13 \mathrm{~Hz}$ a 30Hz. Apresentam-se isoladas, sendo comum a sua coexistência com a banda Gama (Rios \& Glanzmann, 2016). Vem sendo investigada sua participação nos processos de cálculo, tarefas de escolha imediata e supressão de resposta, a exemplo do Paradigma Stroop (Putman, Verkuil, Arias-Garcia, Pantazi \& van Schie, 2014).

O ritmo Alfa $(8 \mathrm{~Hz}$ a $13 \mathrm{~Hz})$ é um dos mais comumente estudado e divulgado. É associado a estados de relaxamento, criatividade e memorização, sendo muito observado em meditações. Em pesquisas, já foi associado ao manejo da ansiedade em programas de biofeedback (Bhat, 2010). 
Já o ritmo Teta atinge freqüências entre $4 \mathrm{~Hz}$ e $8 \mathrm{~Hz}$ e associa-se a estados de consciência rebaixada, nos quais a intuição é potencializada e são feitas conexões emocionais intensas. Uma vez que elimina a ansiedade, relaciona-se a estados de tranqüilidade e maior aproximação do subconsciente, sendo eficaz em técnicas de hipnoterapia (Rios \& Glanzmann, 2016).

As ondas mais lentas são as de freqüência Delta, que variam entre $1 \mathrm{~Hz}$ e $4 \mathrm{~Hz}$ e são capturadas em estados de sono profundo. Sendo praticamente impossível permanecer acordado durante a freqüência Delta, são raras as situações em que esta se apresenta sem que a pessoa esteja dormindo (Travis \& Shear, 2010).

Nas pesquisas na interface religião e atividade cerebral, os resultados aplicativos se ramificam em dois: 1) busca das bases neuroelétricas e 2) isolamento de correlatos neurofuncionais de experiências positivas para empregá-los com fins terapêuticos. Já foi demonstrado, por exemplo, o papel protetivo da religião contra a depressão (Tenke et al.,2013), bem como, a correlação de maiores níveis de convicção religiosa com menores níveis de ansiedade (Good,Inzlicht \& Larson,2014).

Haja vista que estas vivências acarretam maior satisfação com a vida, é necessário investigar os aspectos psicológicos e cognitivos inerentes e, desta maneira, aplicá-los em outros programas e populações. Desta forma, o objetivo desta revisão é fornecer um panorama dos resultados das pesquisas que buscaram compreender os fundamentos neuronais de experiências religiosas e de decorrências do ser religioso através do EEG.

\section{Método}

Os procedimentos metodológicos desta revisão pautaram-se na sistematização de cada etapa do processo de pesquisa. Para tanto, a pesquisa seguiu as recomendações protocolais Preferred Reporting Items for Systematic Reviews and Meta-Analyses (PRISMA) (Galvão, Pansani \&Harrad, 2015), metodologia que preconiza itens e análises preponderantes à sistematizações de literatura.

A busca foi realizada por dois pesquisadores nas bases de dados PubMed/MEDLINE, Science Direct, PsycINFO e Scielo entre os meses de agosto e outubro de 2018, delimitando-se no período entre os anos de 2000 e 2018 e considerando os idiomas português, inglês e espanhol. A sintaxe utilizada como estratégia de busca em todas as bases de dados foram os descritores: "religion" (religião) OR "religious 
experience" (experiência religiosa), individualmente cruzados (AND) com “electroencephalography" (eletroencefalografia) OR "EEG”. No banco de dados Science Direct, ainda, foi utilizado o filtro artigos de pesquisa.

Além do período de publicação e idioma, os estudos desta revisão foram incluídos segundo sua disponibilidade na íntegra, amostra sem recorte de idade em desenvolvimento típico e utilização de EEG como instrumentação. Foram excluídos artigos teóricos, teses, dissertações, cartas ao editor sem dados de pesquisa, capítulos de livro ou livro, estudos que não utilizaram EEG e estudos com amostra de praticantes de yoga e/ou meditações sem cunho religioso relatado.

A consulta das publicações levantadas foi realizada em algumas etapas, seguindo a ordem: (1) exclusão de duplicatas internas e externas de todos os bancos de dados, (2) exclusão por título e (3) exclusão por leitura do resumo. Após o processo descrito, os estudos restantes foram considerados aptos para leitura na íntegra.

O método de extração de dados dos estudos foi alicerçado através de uma planilha que dispunha das seguintes informações: número e gênero dos autores, ano, país e idioma da publicação, tipo de estudo, tamanho da amostra, religião da amostra, idade, técnica de imageamento utilizada, supervisão durante a experiência, pergunta realizada e desfecho do estudo. Por fim, a avaliação do risco de viés dos artigos incluídos na presente revisão foi realizada de forma independente por dois avaliadores, levando em consideração os riscos de distorção nas categorias: viés de seleção e viés de informação.

\section{Resultados}

A busca inicial nos bancos de dados encontrou o total de 866 artigos, dos quais, após a análise dos critérios de inclusão e exclusão e a análise de viés, como descritas na seção referente à metodologia, 23 foram selecionados para compor esta revisão. $\mathrm{O}$ gráfíco de fluxo (Figura 1) pormenoriza os resultados por banco de dados e apresenta o contingente de artigos ao decorrer das etapas de seleção.

Os estudos selecionados, de maneira geral, foram publicados majoritariamente na segunda metade (2009-2018) do período de tempo revisado (2000 - 2018), o que indica uma ascensão do interesse em pesquisas sobre o tema a partir da técnica de EEG. Ressaltam-se números mais elevados de publicações nos anos de 2014 e 2016, seguidos pelo ano de 2013. Estados Unidos, Canadá e Malásia foram os países que mais originaram artigos desta revisão (74\%), que contemplou, ainda, estudos do Reino Unido, Polônia, Japão, Irã, Coréia do Sul e Brasil. 

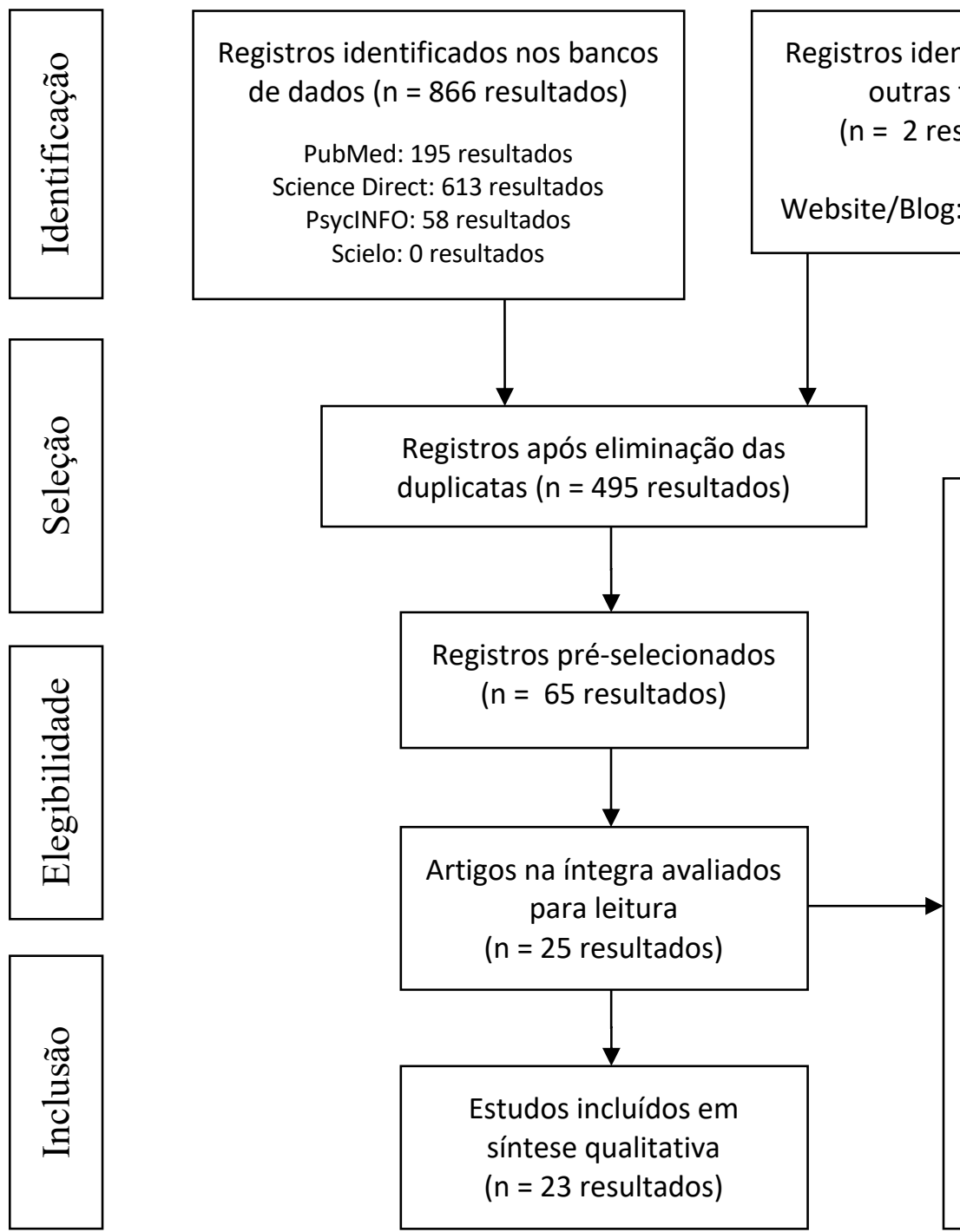
Artigos na íntegra excluídos com justificativa ( $n=40$ resultados) 10 artigos teóricos
19 artigos com amostra de praticantes de yoga e/ou meditações sem cunho religioso definido
6 artigos com amostragem clínica e/ou específica
3 não utilizaram eletroencefalografia (EEG)
2 fora do tema

Figura 1. Etapas de busca e seleção dos artigos

Os artigos selecionados englobam as religiões: Cristianismo, Espiritismo, Budismo e Islamismo, abarcando amostras de 1 a 123 indivíduos. Além disso, algumas das pesquisas não especificam uma única religião, realizando análises comparativas entre grupos de pessoas afiliadas a uma religião, seja ela qual for, e pessoas sem afiliação religiosa e/ou ateus. Os resultados foram sintetizados na Tabela 1. 
Tabela 1.

Principais resultados dos estudos selecionados

\begin{tabular}{|c|c|c|c|c|}
\hline Autores (ano) & Amostra (n) & Religião & Foco & Síntese dos resultados \\
\hline Bastos Jr. et al. (2016) & 20 & Espiritismo & Comunicação mediúnica & $\begin{array}{l}\text { 个ondas Beta, na fase pré-comunicação } \\
\text { 个ondas Beta e Teta, durante a comunicação } \\
\text { 个ondas Beta e Alfa, imediatamente após }\end{array}$ \\
\hline Tenkeet al. (2013) & 52 & Sem especificação & Religião e depressão & 个ondas Alfa \\
\hline $\begin{array}{l}\text { Beauregard \& Paquete } \\
\text { (2008) }\end{array}$ & 14 & Cristianismo & $\begin{array}{l}\text { Variabilidade cortical } \\
\text { associada a estados } \\
\text { místicos }\end{array}$ & $\begin{array}{l}\text { 个ondas Teta e Gama, durante a experiência } \\
\text { mística }\end{array}$ \\
\hline $\begin{array}{l}\text { Thiruchselvametal. } \\
\text { (2017) }\end{array}$ & 49 & Sem especificação & Religião e empatia & $\begin{array}{l}\text { } \text { amplitude de onda em resposta à beleza facial } \\
\text { entre religiosos }\end{array}$ \\
\hline Doufeshet al. (2016) & 20 & Islamismo & Salá(Oração islâmica) & 个ondas Gama nas áreas parietal e frontal \\
\hline Tenkeet al.(2017) & 73 & Sem especificação & Religião e depressão & 个ondas Alfa \\
\hline Vaghefiet al.(2015) & 47 & Islamismo & Alcorão & 个ondas Alfa e Teta \\
\hline Doufeshet al. (2014) & 30 & Islamismo & Salá(Oração islâmica) & 个ondas Alfa nas áreas occipital e parietal \\
\hline Reeves et al. (2014) & 1 & Cristianismo & Glossolalia & $\uparrow$ descargas agudas no lobo temporal direito \\
\hline Doufeshet al.(2012) & 9 & Islamismo & Dhuha(Oração islâmica) & 个ondas Alfa nas áreas parietal e occipital \\
\hline Kamal et al. (2013) & 10 & Islamismo & Alcorão & 个ondas Alfa \\
\hline Delormeet al.(2013) & 6 & Espiritismo & Comunicação mediúnica & $\downarrow_{\text {ondas }}$ Teta na área frontal \\
\hline Inzlitchet al.(2010) & 41 & Sem especificação & Religião e reação ao erro & $\downarrow$ giro do cíngulo \\
\hline Inzlitch et al.(2009) & 28 & Sem especificação & Religião e reação ao erro & $\downarrow$ giro do cíngulo \\
\hline Good et al. (2015) & 123 & Sem especificação & $\begin{array}{l}\text { Religião e reação a } \\
\text { conflitos }\end{array}$ & $\downarrow_{\text {amplitude de onda no giro do cíngulo }}$ \\
\hline $\begin{array}{l}\text { Kossowska et al. } \\
\text { (2016) }\end{array}$ & 42 & Sem especificação & Fundamentalismo religioso & $\downarrow$ amplitude de onda N2 \\
\hline Cho et al. (2018) & 36 & Sem especificação & $\begin{array}{l}\text { Percepção da eficácia de } \\
\text { rituais }\end{array}$ & $\begin{array}{l}\text { 个ondas Beta e Teta, quando em grupo } \\
\downarrow_{\text {ondas Alfa e Beta }}\end{array}$ \\
\hline Oohashiet al.(2002) & 3 & $\begin{array}{c}\text { Kerauhan: Ritual } \\
\text { xamânico balinese }\end{array}$ & Possessão/Transe & 个ondas Teta e Alfa \\
\hline $\begin{array}{l}\text { DeLosAngeles et al. } \\
\text { (2016) }\end{array}$ & 12 & Budismo & Meditação & $\begin{array}{l}\text { 个ondas Alfa e Teta } \\
\downarrow \text { ondas Beta e Gama }\end{array}$ \\
\hline Amihai et al. (2014) & 19 & Sem especificação & $\begin{array}{l}\text { Tradições Theravada e } \\
\text { Vajravana }\end{array}$ & $\begin{array}{l}\text { } \text { ondas Gama, na tradição Theravada } \\
\downarrow \text { ondas Gama, na tradição Vajrayana }\end{array}$ \\
\hline Cahn et al. (2010) & 16 & Sem especificação & Meditação Vipassana & 个ondas Gama \\
\hline Hageman et al. (2010) & 9 & Espiritismo & Comunicação mediúnica & $\downarrow$ ondas Teta nas áreas frontal e temporal \\
\hline Lutz et al. (2004) & 8 & Budismo & Meditação & 个ondas Gama \\
\hline
\end{tabular}

Legenda: $\uparrow$ incremento, $\downarrow$ decréscimo 
O primeiro estudo buscando os correlatos neuroelétricos da experiência religiosa do cristianismo foi realizado por Beauregard e Paquette (2008). Quatorze freiras carmelitas realizaram EEG em uma sala escura e acusticamente isolada durante três momentos: 1) experiência mística (rememorando a experiência mais forte que viveram), 2) controle (rememorando o mais forte estado de união com outro ser humano que viveram) e 3) repouso. Ademais, responderam escalas e instrumentos para análise comportamental.

Neste estudo, as experiências relatadas durante o estado místico foram multidimensionais, implicando mudanças na cognição (representações do eu), percepção (imagens mentais) e emoção (sentimento de paz, amor incondicional). Tais mudanças foram associadas com aumento de ondas Teta nas regiões frontal e parietal, assim como maior relação Teta/Beta nas áreas frontal, central, temporal e parietal. Além disso, também houve um acréscimo nas ondas Gama na área temporal direita e parietal. Os autores apontam que o fato da amostra ter relembrado a mais forte experiência, e não a vivenciado durante a coleta dos dados, seria a principal problemática metodológica, apesar de ter sido uma requisição das próprias participantes. De maneira geral, os resultados sugerem que a experiência mística é mediada por significativas mudanças no sinal EEG.

O fenômeno da glossolalia (ou "falar em línguas"), presente em alguns segmentos religiosos, foi estudado por Reeves, Kose e Abubakr (2014). A amostra consistiu em uma mulher cristã de 44 anos que relatava espasmos musculares no antebraço ao falar em línguas. O sinal EEG não sofreu alteração ao ser capturado em um estado neutro. Após alguns segundos orando em línguas silenciosamente, porém, a participante manifestou descargas agudas no lobo temporal direito, sendo esta frequência significativamente análoga à que se manifesta durante o relaxamento profundo ou sono leve.

Embora os resultados da pesquisa sejam similares a estudos anteriores, muitas são as ressalvas metodológicas apontadas pelos autores. Pelo fato da participante ter achado desrespeitosa a realização das gravações durante o fenômeno, o registro contemplou apenas um momento de oração silenciosa em línguas, sendo ideal a gravação também em voz alta e de maneira espontânea.

O estudo comparativo de Bastos Jr.et al. (2016) investigou a atividade elétrica frontal em mulheres espíritas praticantes da mediunidade e em mulheres espíritas que não 
praticavam a mediunidade. O delineamento do estudo buscou capturar o sinal EEG em três momentos: 1) 30 minutos antes da comunicação, 2) durante a comunicação e 3) imediatamente após a comunicação. Além disso, os participantes responderam escalas e inventários de saúde mental e qualidade de vida. Na fase pré-comunicação, os médiuns apresentaram maior frequência Beta. Alterações dos ritmos Beta e Teta durante a comunicação mediúnica também foram observadas na amostragem mediúnica, assim como aumento dos ritmos Beta e Alfa imediatamente após.

Apesar dos resultados estarem em consonância com outros estudos, algumas limitações devem ser apontadas, como a exclusividade de eletrodos frontais e de mulheres na amostra. Ainda, devido ao equipamento utilizado, a atividade Gama não foi observada, abrindo espaço para extensão e ampliação dos achados.

Os estudos de Delorme etal. (2013) investigaram o sinal EEG de seis médiuns norte americanos. Cada participante realizou duas tarefas: 1) responder vinte e cinco questões a respeito de um falecido sabendo apenas seu nome e 2) experenciar quatro estados mentais: a) Recordação (pensar em uma pessoa viva conhecida pelo médium), b) Percepção (ouvir enquanto um pesquisador descreve características de uma pessoa desconhecida pelo médium), c) Fabricação (imaginar uma pessoa) e d) Comunicação (interagir mentalmente com uma pessoa falecida conhecida a priori).

Em um dos médiuns da amostra, observou-se um decréscimo da freqüência Teta durante os segmentos mediúnicos de alta precisão, sobretudo na área frontal, em consonância com outro estudo com nove médiuns brasileiros (Hageman et al., 2010). Neste mesmo segmento, outro participante apresentou um significativo incremento de ondas Alfa. Para os autores, isto sugere que a comunicação com pessoas falecidas se baseia num cenário mental distinto do utilizado no pensamento e imaginação cotidianos.

A pesquisa de Cho et al. (2018) examinou o correlato neuronal da percepção de religiosos sobre a eficácia de rituais. Para tanto, o desenho da pesquisa baseou-se em duas hipóteses: 1) o grau de excitação do condutor do ritual deve predizer as classificações perceptuais dos religiosos quanto à eficácia do ritual e 2) a co-presença em grupos deve alterar a atenção e o significado atribuído ao ritual. Participaram da pesquisa trinta e seis chineses, que tiveram seus sinais EEG gravados ao assistir vídeos de rituais mediúnicos, tanto individualmente quanto em grupos de três participantes. A combinação de excitação do condutor do ritual elevada e co-presença em grupos mostrou-se mediadora da percepção de religiosos sobre a eficácia ritualística. A atribuição de alta eficácia a rituais 
foi associada com diminuição das atividades Alfa e Beta, sem alterações promovidas pela presença em grupos. Ao mesmo tempo, aqueles que assistiram aos rituais em grupo apresentaram um padrão diferente de sincronização nas freqüências Beta e Teta, comparado a aqueles que assistiram individualmente, o que os autores indicam como uma sintonização de processos cognitivos ocasionada pela presença em grupos sociais.

Um estudo na Indonésia elaborou um método para capturar o sinal EEG durante intensa movimentação, buscando compreender os correlatos neuroelétricos da experiência de possessão e/ou transe (Oohashi et al., 2002). Os pesquisadores propuseram, imediatamente, a análise do sinal EEG durante um ritual de possessão em condições naturais, resguardando-se de possíveis modulações e diferenças causadas no ambiente laboratorial. Apenas um dos três participantes realmente atingiu o estado de possessão e após análise de espectro de sinal, identificou-se um incremento nas atividades Teta e Alfa. Muito embora o estudo faça uma extensa análise dos resultados e realize comparações com estados patológicos e distúrbios mentais, os resultados ainda são insuficientes para a compreensão da neurofisiologia e neuroanatomia durante a dissociação ritualística.

Uma gama de pesquisas buscando compreender a ativação cortical decorrente de orações e rituais islâmicos vem sendo realizada (Doufesh et al., 2012; Vaghefi et al., 2015). Propõe-se que práticas muçulmanas podem produzir maiores estados de calma e acréscimo na concentração e autoconsciência, diferenciando-se de algumas orações cristãs e meditações budistas por envolver movimentos corporais e posições como parte da experiência.

Doufeshet al. (2012), por exemplo, examinou a densidade espectral de energia (Power SpectralDensity- PSD) da frequência Alfa durante a performance da oração Dhuha. Diferentemente da oração Salá, que é obrigatória a todos os muçulmanos, Dhuha é voluntária e tem o propósito de expressar gratidão ao novo dia e apelo à boa sorte, geralmente praticada pela manhã.A amostra total foi de nove muçulmanos, sendo seis homens e três mulheres. O delineamento da pesquisa abarcou a captura do espectro de sinal Alfa em três sessões: 1) sentado em uma cadeira em repouso e com os olhos abertos por 1 minuto, 2) performando quatro ciclos da oração Dhuha e 3) performando quatro ciclos dos movimentos da oração sem recitar os versos do Alcorão correspondentes. Os ciclos de oração contam com a performance de algumas posições, a saber: de pé, 
curvando-se, de pé, prostrado, sentado, prostrado e sentado. Para informações visuais sobre as posições, consultar imagens disponibilizadas no artigo original.

Os resultados deste estudo apontaram para um significativo aumento no espectro de sinal Alfa durante a posição prostrada, em que a cabeça toca o chão. Durante a prostração, observa-se maior estado de calma e concentração e redução da tensão, o que confirma resultados anteriores que correlacionam a frequência Alfa com estados de mente relaxados.

Posteriormente, os mesmos autores realizaram um estudo investigando o efeito da oração Salá no espectro de sinal Alfa e sua relação com o sistema nervoso autônomo (Doufeshet al. 2014). Trinta muçulmanos participaram do estudo e gravaram o sinal EEG em três momentos: 1) antes da oração, 2) durante a prática e 3) imediatamente após. A análise dos resultados mostrou diferenças significativas entre as capturas antes e durante a oração em todos os eletrodos configurados. Entretanto, não houve diferença significativa entre as capturas durante a prática e imediatamente após, o que pode sugerir uma continuidade da frequência gerada durante a oração. Demarcou-se, sobretudo, incremento da frequência Alfa nas áreas occipital e parietal.

Os correlatos neuroelétricos da oração Salá foram reanalisados por Doufesh et al. (2016), com enfoque na freqüência Gama. Participaram do estudo vinte mulçumanos que tiveram o sinal EEG capturado em quatro sessões: 1) sentado e relaxado por três minutos, 2) performance da Salá, 3) performance apenas dos movimentos da Salá e 4) imediatamente após, sentado e relaxado.Os resultados mostraram que a intensidade da frequência Gama foi maior durante a performance completa da Salá comparada à performance apenas dos movimentos, especificamente nas áreas frontal e parietal. A frequência Gama mostrou-se maior, também, nas posições prostrado e curvando-se.

Um estudo de 2015 buscou compreender o efeito do Alcorão em uma amostra de quarenta e sete mulçumanos persas (Vaghefi et al., 2015). Para tanto, os voluntários ouviram a dois tipos de texto: 1) Alcorão e 2) outros textos sem significado sacro, de maneira consciente (sabendo a priori se o texto do áudio fazia ou não parte do Alcorão) e não consciente. Ouvir ao Alcorão de maneira consciente foi positivamente correlacionado com um significativo incremento da freqüência Teta na maioria das regiões cerebrais, comparado às outras condições. Ouvir ao Alcorão sem saber disso, ainda, gerou um aumento da freqüência Alfa no lobo frontal, confirmando resultados anteriores. 
O efeito da leitura do Alcorão também foi investigado por Kamal, Mahmood e Zakaria (2013). Com uma amostra de dez participantes, o objetivo da pesquisa era comparar o antes, o durante e o depois da leitura do Alcorão e da leitura de livros sem significado sagrado. Os resultados apontaram para um acréscimo da freqüência alfa durante a leitura do Alcorão, bem como foi notável o decréscimo da mesma frequência durante a leitura de outros escritos. Dessa forma, observando a literatura já existente na área e os próprios resultados, os autores sugerem que a leitura do Alcorão pode promover estados de mente relaxados e níveis atencionais mais elevados.

As últimas décadas vêm presenciando uma ascensão do interesse nas pesquisas sobre os correlatos neuronais de diferentes técnicas de meditação. Contudo, é muito freqüente estudos com praticantes de meditação que não se consideram religiosos ou seguidores de paradigmas religiosos. Por este motivo, muitos desses estudos não foram contemplados pelos critérios de inclusão desta revisão. Dentre as muitas pesquisas, DeLosAngeles et al. (2016) buscaram as correlações eletroencefalográficas de cinco estados da meditação budista. Para tal, doze budistas experientes participaram do estudo. As conclusões indicaram que os praticantes experientes de meditação atingem estados cerebrais diferentes dos atingidos pelo grupo controle, e que nos experientes as mudanças neuronais se intensificam conforme sucedem-se estados mais profundos de meditação. Nos que meditavam, houve acréscimo na freqüência Alfa e Teta, assim como decréscimo nas freqüências Beta e Gama.

Outro estudo comparou os correlatos neurofisiológicos e cognitivos de duas diferentes tradições: Theravada e Vajrayana (Amihai \& Kozhevnikov, 2014). Os resultados do primeiro estudo mostraram que as meditações da tradição Theravada produziram ativação parassimpática aumentada, indicando uma resposta de relaxamento. Em contraste, meditações da tradição Vajrayana produziram ativação simpática, indicativa de excitação. Os resultados do segundo estudo explicitaram um aumento significativo no desempenho em tarefas cognitivas nos meditadores da tradição Vajrayana, indicando uma fase de alerta aprimorada, possivelmente devido à excitação. Ainda, os sinais EEG demonstraram aumento da freqüência Gama na prática de tradição Theravada, em consonância com outros estudos (Cahn, Delorme \& Polich, 2010), em oposição, diminuição da freqüência Gama nas técnicas da tradição Vajrayana.

Lutz et al. (2004) capturaram os sinais EEG de oito budistas que praticavam a meditação há mais de 15 anos. Os resultados apontaram que meditadores experientes 
auto-induzem a frequência Gama durante a meditação, atingindo estados de autocontrole, percepção unificada da realidade e vinculação sensorial.

Por fim, há estudos sem o enquadre de religiões ou segmentos específicos, averiguando possíveis diferenças no sustentáculo neuronal de pessoas religiosas, de modo geral. Para isto, os pesquisadores propõem comparações entre grupos de religiosos e não religiosos e, também, entre religiosos e ateus. Thiruchselvam et al. (2017) examinaram se indivíduos não religiosos seriam menos sensíveis à empatia ("social conformity"), construindo o desenho da pesquisa a partir de um paradigma de julgamento de beleza facial. Participaram da amostra quarenta e nove pessoas divididas em dois grupos, um de vinte e quatro não religiosos e outro de vinte e cinco religiosos. Nas medidas de autorelato, não houve diferença significativa entre os grupos. Entretanto, os sinais emitidos no EEG em resposta à beleza facial entre não religiosos mostraram-se menos sensíveis à influência dos pares, comparado ao grupo de religiosos.

Estudos anteriores mostraram diferenças no plano de fundo cerebral entre indivíduos religiosos e não religiosos. Em particular, foi feito um estudo com aqueles com sólida convicção religiosa (Inzlicht et al., 2009). Os autores sugeriram que a convicção religiosa é marcada pela reatividade reduzida no córtex cingulado, sistema envolvido na experiência da ansiedade e importante para o autocontrole. Neste grupo, a atividade neural emitida em resposta ao erro, medida através do Paradigma Stroop, mostrou-se inferior à emitida pelo grupo de não religiosos. Em estudos posteriores sugere-se uma ligação causal entre a religião e a maneira como o cérebro processa a reação ao erro e conflitos (Inzlitch et al. 2010; Good et al. 2015).

Apesar dos resultados solidamente descritos, Kossowska et al. (2016) apontam a falta da análise do aspecto fundamentalismo religioso nas pesquisas de Inzlicht et al. (2009) e realizam uma pesquisa baseada nesta investigação. A partir da hipótese do estudo, esperava-se que maiores índices de fundamentalismo religioso estariam associados com respostas neuronais menos ansiogênicas à incerteza, ao desconhecido. A amostra do estudo contou com quarenta e dois estudantes que responderam a escalas de religiosidade e realizaram a captura dos sinais EEG. Os resultados sugeriram que o fundamentalismo religioso modera os aspectos afetivos do monitoramento do desempenho sob a incerteza, apesar de não moderar o desempenho comportamental real, uma vez que os mesmos participantes apresentaram vigilância típica em um contexto neutro. 
Tenke et. al. (2013) investigaram se indivíduos religiosos teriam maior fator protetivo contra a depressão e se estes apresentariam maior ritmo Alfa. Os resultados da pesquisa indicaram, entre outros, que dos cinqüenta e dois participantes, os que pontuaram maiores níveis de espiritualidade na escala utilizada apresentaram maior amplitude Alfa comparados aos que obtiveram níveis mais baixos.Vinte anos após os estudos iniciais, os mesmos autores replicaram e ampliaram o escopo da pesquisa (Tenke et al., 2017). Setenta e três indivíduos participaram da amostra, sendo vinte e um destes já participantes das pesquisas anteriores dos autores. O novo estudo foi baseado em duas hipóteses: 1) aqueles que foram inicialmente identificados com alta religiosidade/espiritualidade continuariam a apresentar a frequência Alfa elevada e 2) aqueles que aumentaram e subsequentemente manteram seus índices de religiosidade também mostrariam alta freqüência Alfa. Embora a hipótese 1 tenha sido confirmada, os participantes que alteraram seus níveis de religiosidade ao decorrer da pesquisa apresentaram menor freqüência Alfa, negando a hipótese 2.

\section{Discussão}

Como já mencionado, um dos grandes entraves para que as pesquisas da área comecem a receber mais atenção é o ainda muito marcado antagonismo criado entre ciência e religião. Soma-se à notabilidade do tema, o fato de serem poucas as publicações que abarcam o assunto, especialmente no Brasil. Investigar cientificamente os aspectos de influência da atividade religiosa sobre processos neurológicos inspira possibilidades, considerando as já descritas evidências de modificações fisiológicas, na configuração mente-corpo, na motivação e em perspectivas psicológicas. Além disso, os estudos sobre o fenômeno religioso raramente se distanciaram das questões que envolvem a consciência e os estados mentais superiores (Cescon, 2011).

Sabe-se que o cristianismo é uma das religiões de maior propagação nas sociedades ocidentais, contudo, não são muitos os estudos que buscam compreender os fenômenos a ele associado. Os resultados de Beauregard e Paquete (2008), ainda assim, podem sugerir que rituais cristãos se associam a estados de percepção potencializada, nos quais são feitas integração sensorial e conexões emocionais profundas, descritos pelo aumento das bandas Gama e Teta.

Os estudos da glossolalia, um dos fenômenos religiosos que mais chamam atenção, continuam incipientes e suscetíveis a interpretações metodológicas. Embora a 
relação entre a atividade do lobo temporal e a experiência religiosa tenha sido apontada na literatura antes mesmo dos experimentos com o capacete de Persinger (1987), ainda não há consenso entre os pesquisadores. Autores que estudam o tema sugerem inúmeras limitações e recebem críticas defendendo que, de uma sensação produzida em condições laboratoriais, não se pode inferir grandes conclusões. Ainda, a manifestação do lobo temporal durante a glossolalia pode ser produzida pelo próprio fenômeno, e não necessariamente sua etiologia, o que demanda maior aprofundamento para melhor compreensão.

Os resultados dos estudos com médiuns (Bastos Jr. et al., 2014; Delorme et al., 2013) reforçam a clássica hipótese de absorção, mostrando aumento da atenção concentrada concomitante a uma diminuição da auto-consciência durante comunicações mediúnicas. Além disso, a recorrente diminuição da atividade Teta durante comunicações mediúnicas pode sugerir um estado de meditação profunda acompanhado de um sentimento de conexão emocional e unidade com o universo (Hageman et al., 2010; Delorme et al., 2013).

Esses autores sugerem que, justamente no limite entre Alfa e Teta $(7 \mathrm{~Hz}$ e $8 \mathrm{~Hz})$ encaixam-se as possibilidades de neuroprogramação, assim como é a condição ideal para técnicas com visualização e uso da criatividade. É neste ínterim, quando o corpo se encontra em relaxamento profundo, mas ainda há consciência do ambiente, que se cria a contingência necessária para conscientemente modificar esquemas da realidade (Bastos Jr. et al., 2014; Hageman et al., 2010).

Também, a possessão ritualística foi associada ao incremento da ativação Teta (Oohashi et al., 2002). Esta frequência estaria relacionada com inibição de respostas, sobretudo em situações onde a pessoa tenta ativamente reprimi-la (Kirmizi-Alsan et al., 2006), como em tarefas do Paradigma Go/No-Go. É capturada durante os sonhos e o sono $R E M$, sendo relacionada, também, a retenção da memória.

As pesquisas interessadas em compreender os correlatos neuronais dos rituais e fenômenos do Islamismo apontam, dentre outros, o acréscimo na freqüência Alfa (Vaghefi et al.,2015; Doufesh et al., 2012; Doufesh et al., 2014; Kamal et al., 2013) e modificações significativas nas freqüências Gama e Teta (Doufesh et al., 2016). Essas pesquisas indicam, também, correlações positivas entre medidas atencionais e mnemônicas e práticas islâmicas. 
As ondas Alfa estão presentes quando a pessoa se encontra em relaxamento profundo e, via de regra, de olhos fechados. A produção dessas ondas é promovida normalmente pelo sistema parassimpático, com supressão do simpático (Murata et al., 2004). Este dado vai de encontro com a desaceleração dos batimentos cardíacos e a diminuição da pressão arterial, promovidos pelas posições e movimentos ritualísticos.

De modo geral, os estudos sobre as meditações budistas sugerem incremento na atividade Gama, Alfa e Teta (Lutz et al., 2004; DeLosAngeles et al., 2016). Apesar disso, outros achados indicam diferenças nos correlatos neuronais de diferentes tradições (Amihai\& Kozhevnikov, 2014), que se manifestam através da modulação da freqüência Gama.

As técnicas da tradição Vajrayana foram associadas a um aumento do desempenho cognitivo (incremento Alfa, redução Gama). Já a tradição Theravada engendrou mecanismos de relaxamento e atenção fluida, não depositada (incremento Gama). Para os autores, esses resultados são consistentes com as escrituras tibetanas que descreviam as técnicas Theravada como aquelas que acalmam e relaxam a mente, e técnicas Vajrayana como aquelas que requerem "um despertar da qualidade da mente", mostrando que as meditações Theravada e Vajrayana são baseadas em diferentes mecanismos, que dão origem a uma resposta de relaxamento ou de excitação (Amihai\& Kozhevnikov, 2014).

Vem sendo proposto que a frequência Gama tem importante participação na experimentação consciente e nos processos de consciência. Beauregard e Paquete (2008) sugerem que, a partir dos estudos realizados com EEG, meditações e experiências religiosas, é razoável depreender que o aumento da atividade Gama durante experiências religiosas estaria relacionado com a experimentação subjetiva de união com Deus.

São volumosas as pesquisas realizadas nesta interface que buscam a compreensão acerca dos correlatos do ser religioso, sem definição de uma religião específica. Dentre os achados mais significativos, o aumento da freqüência Alfa se destaca (Tenke et al., 2013; Tenke et al., 2017), seguido de incremento na freqüência Beta (Cho et al., 2018). De acordo com pesquisas recentes, a estimulação com freqüência Beta mostrou ser eficaz no tratamento da fadiga e de problemas de aprendizagem e concentração. Ainda, foi confirmada a melhora na acuidade visual e coordenação, eficácia no tratamento de dislexia e falta de concentração e ganho de QI na faixa de 8 a 10 pontos (Rios \& Glanzmann, 2016). 
Como discutido, desde contextos bem enquadrados até situações rotineiras, religiosos demonstram acessar com maior facilidade configurações neuronais que subvencionam respostas de calmaria e unificação sensorial. Dente outros, a religião foi positivamente correlacionada a menores índices de depressão (Tenke et al., 2013), menor ansiedade (Good et al., 2015), maior empatia (Thiruchselvam et al., 2017) e melhor desempenho atencional e mnemônico (Doufesh et al., 2016).

Desta maneira, conhecer o plano de fundo neural dessa forma de experenciar o mundo, as relações e o eu pode oferecer instrumentos que apliquem - como uma forma de engenharia reversa - os benefícios do ser religioso, não necessariamente nas mesmas situações que originaram tais favorecimentos. Esta área de interseção aspira práticas qualificadas tanto no campo cognitivo, quanto no psicológico e emocional, desabrochando um horizonte promissor.

Exemplo disto, a atual pesquisa conduzida por Davidson (2007) vem tentando entender a eficácia da meditação em determinar dois efeitos: redução do efeito do envelhecimento cortical e aumento das habilidades cognitivas dependentes da atenção. Rios e Glanzmann (2016) desenvolvem uma tecnologia com objetivo de ativar estados de consciência por meio de estimulação de ondas cerebrais, usando sons binaurais disponível em um aplicativo. Dentre os objetivos da pesquisa, o uso da tecnologia computacional visa promover modificações em estados de estresse.

Ainda, além do uso de estimulação de ondas cerebrais para tratamento de adicções e ansiedade, destacam-se também os estudos que visam o controle da dor e sono (Wilson, 1990; Rhodes, 1993), o acesso a estados anômalos de consciência (Atwater, 1997) e o efeito antinociceptivo durante a anestesia (Kliempt et al., 1999). Assim sendo, por estar explícita na literatura uma gama de benefícios acarretados por essa tecnologia, é preciso salientar a relevância desses estudos e fomentar a disseminação desses conhecimentos e técnicas.

Algumas limitações devem ser apontadas no presente manuscrito. Primeiro, os critérios incluem apenas estudos com EEG, não abarcando aqueles que utilizaram métodos outros, como os de neuroimagem (sMRI, fMRI, SPECT, PET). Segundo, a análise dos resultados focou na compreensão da modificação das freqüências e amplitudes de ondas cerebrais, de maneira que análises com técnicas derivadas do EEG não foram esmiuçadas da mesma forma. Dentre estas, destacam-se os potenciais elétricos relacionados a eventos (ERPs), técnica que busca relacionar a reação elétrica a uma 
operação cognitiva específica, muito usada na neurociência cognitiva e psicologia cognitiva.

\section{Considerações finais}

O objetivo dessa revisão sistemática foi compilar os resultados das pesquisas que buscaram compreender os fundamentos neuronais das experiências e ritos religiosos e das decorrências do ser religioso a longo-prazo. Dada a multiplicidade de técnicas e instrumentos utilizados, optou-se pela seleção dos estudos realizados através de Eletroencefalograma, haja vista a recorrência destes na literatura.

Dentre os resultados, a modulação das ondas Gama foi observada nos rituais cristãos e budistas, as ondas Teta apresentaram aumento no Cristianismo e diminuição no Espiritismo e as ondas Alfa nos rituais islâmicos. Os pesquisadores sugerem que esses resultados podem estar correlacionados com maior senso de propósito e significado de vida, bem como amplificação da empatia e compaixão.

Também, comparando religiosos e não religiosos e/ou ateus, religiosos demonstraram acessar com maior facilidade configurações neuronais promotoras de calmaria e unificação sensorial. Dente outros, a religião foi positivamente correlacionada com menores índices de depressão, menor ansiedade, maior empatia e melhor desempenho atencional e mnemônico.

Ciência e religião não são, afinal, homólogas, mas convergem no momento em que explicam complementarmente fenômenos que não se deixam explicar de modo exaustivo por só uma delas, fornecendo um caráter de atualidade e ineditismo. Demarcase a importância da divulgação científica válida, especialmente por se tratar de um aspecto atentado no campo. Esta área de interseção aspira práticas qualificadas tanto no campo cognitivo, quanto no psicológico e emocional, desabrochando um horizonte promissor.

\section{Referências}

Amihai, I., \& Kozhevnikov, M. (2014). Arousal vs. relaxation: a comparison of the neurophysiological and cognitive correlates of Vajrayana and Theravada meditative practices. PloS one, 9(7), e102990. doi:10.1371/journal.pone.0102990 
Atwater, F.H. (1997). Accessing anomalous states of consciousness with a binaural beat technology. Journal of Scientific Exploration, 11(3), pp. 263-274. Recuperado dehttps://www.gohaven.org/uploads/6/9/8/2/69821929/hemisync anomalous_state s.pdf

Bastos Jr., M. A. V., Bastos, P. R. H. D. O., Osório, I. H. S., Muass, K. A. R. C., Iandoli Jr, D., \& Lucchetti, G. (2016). Frontal electroencephalographic (EEG) activity and mediumship: a comparative study between spiritist mediums and controls. Archives of Clinical Psychiatry (São Paulo), 43(2), 20-26. doi: 10.1590/0101-60830000000076

Beauregard, M., \& Paquette, V. (2008). EEG activity in Carmelite nuns during a mystical experience. Neuroscience Letters, 444(1), 1-4. doi: 10.1016/j.neulet.2008.08.028

Bhat, P. (2010). Efficacy of Alfa EEG wave biofeedback in the management of anxiety. Industrial Psychiatry Journal,19(2),111-114. doi: 10.4103/0972-6748.90341

Cahn, B. R., Delorme, A., \& Polich, J. (2010). Occipital gamma activation during Vipassana meditation. Cognitive processing, 11(1), 39-56. doi: 10.1007/s10339-0090352-1

Cescon, E. (2011). Neurociência e religião: as pesquisas neurológicas em torno da experiência religiosa. Estudos de religião, 25(41), 77-96. doi: 10.15603/21761078/er.v25n41p77-96

Cho, P. S., Escoffier, N., Mao, Y., Ching, A., Green, C., Jong, J., \& Whitehouse, H. (2018). Groups and Emotional Arousal Mediate Neural Synchrony and Perceived Ritual Efficacy. Frontiers in Psychology, 9, 2071. doi: 10.3389/fpsyg.2018.02071

da Mota Gomes, M. (2015). Bases fisiológicas do eletroencefalograma. Revista Brasileira de Neurologia,51(1), 12-17.Recuperado de http://files.bvs.br/upload/S/0101-8469/2015/v51n1/a4729.pdf

Delorme, A., Beischel, J., Michel, L., Boccuzzi, M., Radin, D., \& Mills, P. (2013). Electrocortical activity associated with subjective communication with the deceased. Frontiers in psychology, 4, 834, 1-10. doi: 10.3389/fpsyg.2013.00834

DeLosAngeles, D., Williams, G., Burston, J., Fitzgibbon, S. P., Lewis, T. W., Grummett, T. S. \& Willoughby, J. O. (2016). Electroencephalographic correlates of states of concentrative meditation. International Journal of Psychophysiology, 110, 27-39. doi: 10.1016/j.ijpsycho.2016.09.020 
Doufesh, H., Faisal, T., Lim, K. S., \& Ibrahim, F. (2012). EEG spectral analysis on Muslim prayers. Applied psychophysiology and biofeedback,37(1), 11-18. doi: 10.1007/s10484-011-9170-1

Doufesh, H., Ibrahim, F., Ismail, N. A., \& Wan Ahmad, W. A. (2014). Effect of Muslim prayer (Salat) on $\alpha$ electroencephalography and its relationship with autonomic nervous system activity. The Journal of Alternative and Complementary Medicine, 20(7), 558-562. doi: 10.1089/acm.2013.0426

Doufesh, H., Ibrahim, F., \& Safari, M. (2016). Effects of Muslims praying (Salat) on EEG gamma activity. Complementary therapies in clinical practice, 24, 6-10. doi: 10.1016/j.ctcp.2016.04.004

Galvão, T. F., Pansani, T. D. S. A., \& Harrad, D. (2015). Principais itens para relatar revisões sistemáticas e meta-análises: a recomendação PRISMA. Epidemiologia e Serviços de Saúde, 24 (2), 335-342. doi: 10.5123/S1679-49742015000200017

Good, M., Inzlicht, M., \& Larson, M. J. (2015). God will forgive: reflecting on God's love decreases neurophysiological responses to errors. Social cognitive and affective neuroscience, 10(3), 357-363. doi: 10.1093/scan/nsu096

Hageman, J. H., Peres, J. F. P., Moreira-Almeida, A., Caixeta, L., Wickramasekera, I. I.,\& Krippner, S. (2010). The neurobiology of trance and mediumship in Brazil. In S. Krippner \& H. L. Friedman (Eds.) Mysterious minds: The neurobiology of psychics, mediums, and other extraordinary people(pp. 85-111). Santa Barbara, CA: ABCCLIO.

Inzlicht, M., McGregor, I., Hirsh, J. B., \& Nash, K. (2009). Neural markers of religious conviction. Psychological Science,20(3), 385-392. doi: 10.1111/j.14679280.2009.02305.x

Inzlicht, M., \& Tullett, A. M. (2010). Reflecting on God: Religious primes can reduce neurophysiological response to errors. Psychological Science, 21(8), 1184-1190. doi: $10.1177 / 0956797610375451$

Kamal, N. F., Mahmood, N. H., \& Zakaria, N. A. (2013). Modeling brain activities during reading working memory task: Comparison between reciting Quran and reading book. Procedia-Social and Behavioral Sciences,97 (6), 83-89. doi: 10.1016/j.sbspro.2013.10.207 
Kirmizi-Alsan, E., Bayraktaroglu, Z., Gurvit, H., Keskin, Y. H., Emre, M., \& Demiralp, T. (2006). Comparative analysis of event-related potentials during Go/NoGo and CPT: decomposition of electrophysiological markers of response inhibition and sustained attention. Brain research, 1104(1), 114-128. doi: 10.1016/j.brainres.2006.03.010

Kliempt, P., Ruta, D., Ogston, S., Landeck, A., \& Martay, K. (1999). Hemisphericsynchronization during anesthesia: a double-blind randomized trial using audiotapes for intra-operative nociception control. Anesthesia, 54, 769-773. doi: 10.1046/j.1365-2044.1999.00958.x

Kossowska, M., Szwed, P., Wronka, E., Czarnek, G., \& Wyczesany, M. (2016). Anxiolytic function of fundamentalist beliefs: neurocognitive evidence. Personality and Individual Differences, 101, 390-395. doi: 10.1016/j.paid.2016.06.039

Luft, C., \& Andrade, A. (2006). A pesquisa com EEG aplicada à área de aprendizagem motora. Revista Portuguesa de Ciências do Desporto, 6(1), 106-115. Recuperado de http://www.scielo.mec.pt/pdf/rpcd/v6n1/v6n1a12.pdf

Lutz, A., Greischar, L. L., Rawlings, N. B., Ricard, M., \& Davidson, R. J. (2004). Longterm meditators self-induce high-amplitude gamma synchrony during mental practice. Proceedings of the national Academy of Sciences, 101(46), 16369-16373. doi:10.1073/pnas.0407401101

Murata, T., Takahashi, T., Hamada, T., Omori, M., Kosaka, H., Yoshida, H., \& Wada, Y.(2004). Individual trait anxiety levels characterizing the properties of Zen meditation. Neuropsychobiology, 50(2), 189-194. doi: 10.1159/000079113

Oohashi, T., Kawai, N., Honda, M., Nakamura, S., Morimoto, M., Nishina, E., \& Maekawa, T. (2002). Electroencephalographic measurement of possession trance in the field. Clinical Neurophysiology,113(3), 435-445. doi: 10.1016/S13882457(02)00002-0

Persinger, M. A. (1987). Neuropsychological bases of God beliefs. New York: Praeger Publishers.

Putman, P., Verkuil, B., Arias-Garcia, E., Pantazi, I., \& van Schie, C. (2014). EEG theta/beta ratio as a potential biomarker for attentional control and resilience against deleterious effects of stress on attention. Cognitive, Affective, \& Behavioral Neuroscience, 14(2), 782-791. doi: 10.3758/s13415-013-0238-7 
Reeves, R. R., Kose, S., \& Abubakr, A. (2014). Temporal lobe discharges and glossolalia. Neurocase, 20(2), 236-240. doi: 10.1080/13554794.2013.770874

Rhodes, L. (1993). Use of the Hemi-Sync super sleep tape with a preschool-aged child.

Hemi-Sync Journal, XI(4), pp. iv-v.Recuperado de https://www.monroeinstitute.org/article/2721

Sabbatini,R. M. E.(1997). História do Eletroencefalograma. Mapeando o cérebro. Revista cérebro e mente, 3, set/nov. Recuperado de http://www.cerebromente.org.br/n03/tecnologia/historia_p.htm

Rios, L. M., \& Glanzmann, J. H. (2016). Aplicativo que manipula Ondas Cerebrais por meio de Frequências Binaurais. Seminários de Trabalho de Conclusão de Curso do Bacharelado em Sistemas de Informação, 1(1). Recuperado de http://periodicos.jf.ifsudestemg.edu.br/revistabsi/article/view/31/29

Tenke, C. E., Kayser, J., Miller, L., Warner, V., Wickramaratne, P., Weissman, M. M., \& Bruder, G. E. (2013). Neuronal generators of posterior EEG alpha reflect individual differences in prioritizing personal spirituality. Biological psychology, 94(2), 426-432. doi: 10.1016/j.biopsycho.2013.08.001

Tenke, C. E., Kayser, J., Svob, C., Miller, L., Alvarenga, J. E., Abraham, K. \& Bruder, G. E. (2017). Association of posterior EEG alpha with prioritization of religion or spirituality: a replication and extension at 20-year follow-up. Biological psychology, 124, 79-86. doi: 10.1016/j.biopsycho.2017.01.005

Thiruchselvam, R., Gopi, Y., Kilekwang, L., Harper, J., \& Gross, J. J. (2017). In God we trust? Neural measures reveal lower social conformity among non-religious individuals. Social cognitive and affective neuroscience, 12(6), 956-964. doi: $10.1093 /$ scan/nsx023

Travis, F., \& Shear, J. (2010). Focused attention, open monitoring and automatic selftranscending: categories to organize meditations from Vedic, Buddhist and Chinese traditions. Consciousness and cognition, 19(4), 1110-1118. doi: 10.1016/j.concog.2010.01.007

Trudeau, D. L. (2000). The treatment of addictive disorders by brain wave biofeedback: a review and suggestions for future research.Clinical Electroencephalography, 31(1),13-22. doi: 10.1177/155005940003100107 
Vaghefi, M., Nasrabadi, A. M., Golpayegani, S. M. R. H., Mohammadi, M. R., \& Gharibzadeh, S. (2015). Spirituality and brain waves. Journal of medical engineering \& technology, 39(2), 153-158. doi: 10.3109/03091902.2014.1001528

Valle, E. (2001). Neurociências e religião: interfaces. Revista de estudos da religião, 6(3), 1-46. Recuperado de https://www.pucsp.br/rever/rv3_2001/p_valle.pdf

Varela, F., Lachaux, J. P., Rodriguez, E., \& Martinerie, J. (2001). The brainweb: Phase synchronization and large-scale integration. Natural Review of Neuroscience, 2(4), 229-239. doi: 10.1038/35067550

von Stein, A., \& Sarnthein, J. (2000). EEG frequency and the size of cognitive neuronal assemblies. Behavioral and Brain Sciences, 23(3), 413-414. doi: https://doi.org/10.1017/S0140525X0038325X

Wilson, E.S. (1990). Preliminary study of the Hemi-Sync sleep processor. ColoradoAssociation for Psychophysiologic Research. 